Hiroyasu Iwasa • Masahiko Kurabayashi • Ryozo Nagai

Yusuke Nakamura • Toshihiro Tanaka

\title{
Multiple single-nucleotide polymorphisms (SNPs) in the Japanese population in six candidate genes for long QT syndrome
}

\begin{abstract}
We report here 20 single-nucleotide polymorphisms (SNPs), including 15 novel ones, in six genes that are considered to be candidates for long QT syndrome (LQTS): 2 SNPs in $K C N B 1,3$ in $K C N D 3,3$ in $K C N J 11,7$ in $A B C C 9$, 3 in $A D R B 1$, and 2 in $S L C 18 A 2$. We also examined their allelic frequencies in a Japanese sample population of LQTS-affected and nonaffected individuals. These data will be useful for genetic association studies designed to investigate acquired arrhythmias.
\end{abstract}

Key words Long QT syndrome · Single-nucleotide polymorphism - Japanese population - Cardiac potassium channel · $\beta 1$-Adrenergic receptor - Vesicular monoamine transporter

\section{Introduction}

Long QT syndrome (LQTS), an arrhythmogenic disorder characterized by prolongation of the QT interval on electrocardiograms (ECGs), often causes syncope or sudden cardiac death as a result of recurrent and lethal arrhythmias. Ventricular tachycardia, torsades de pointes, and ventricular fibrillation are among the manifestations of LQTS. Five genes in which inherited mutations are responsible for this syndrome have been identified to date: $K C N Q 1$ (KVLQT1), KCNH2 (HERG), KCNE1, KCNE2, and SCN5A (Bennett et al. 1995; Curran et al. 1995; Wang et al. 1996a,b; Splawski et al. 1997; Abbott et al. 1999). A mutant

H. Iwasa $\cdot$ Y. Nakamura $\cdot$ T. Tanaka $(\bowtie)$

Laboratory of Molecular Medicine, Human Genome Center,

Institute of Medical Science, University of Tokyo, 4-6-1

Shirokanedai, Minato-ku, Tokyo 108-8639, Japan

Tel. +81-3-5449-5373; Fax +81-3-5449-5406

e-mail: toshitan@ims.u-tokyo.ac.jp

H. Iwasa $\cdot$ M. Kurabayashi

Second Department of Internal Medicine, Gunma University School of Medicine, Gunma, Japan

R. Nagai

Department of Cardiovascular Medicine, Graduate School of

Medicine, University of Tokyo, Tokyo, Japan form of one of these five genes can be detected in nearly $40 \%$ of LQTS families, but the abnormalities responsible for the syndrome in the other $60 \%$ appear to be associated with other, unknown genes.

The pathogenesis of QT prolongation has been revealed only in part, and natural variations in cardiac ion channels are thought to be strong candidates for association with the disorder. For example, Kv2.1 and Kv4.3 are pore-forming subunits of cardiac potassium channels; pharmacological studies in transgenic animals have suggested that abnormalities in these channels might contribute to QT prolongation (Barry et al. 1998; Xu et al. 1999). $\mathrm{K}_{\text {ATP }}$ channels (Kir6.2/SUR2A) are also highly expressed in the heart (Noma 1983); they are the molecular targets of nicorandil (Hiraoka and Fan 1989), an efficient drug for treating LQTS (Fujimoto et al. 1999).

On the other hand, as sympathetic denervation of the left side of the heart can sometimes prevent sudden cardiac death in LQTS patients (Bhandari et al. 1984), it is also important to consider molecules that participate in the function of sympathetic nerves. For example, the $\beta 1$-adrenergic receptor is the target molecule of $\beta$-blockers; and heterozygous deficiency of $S L C 18 A 2$, the gene encoding vesicular monoamine transporter (VMAT2), can induce a prolonged QT interval and sudden death in knockout mice (Itokawa et al. 1999).

Here, we report our finding of multiple single-nucleotide polymorphisms (SNPs) in six candidate genes for LQTS (KCNB1, encoding Kv2.1; KCND3, encoding Kv4.3; $K C N J 11$, encoding Kir6.2; $A B C C 9$, encoding SUR2A; $A D R B 1$, encoding $\beta 1$-adrenergic receptor; and SLC18A2, encoding VMAT2), along with their allelic frequencies in normal and LQTS-affected Japanese subjects.

\section{Subjects and methods}

In the course of a search for mutations in six candidate genes in Japanese LQTS patients, we identified 20 SNPs that were present in the genomic DNA of 104 LQTS 
Table 1. PCR primers used to cover the coding regions of $K C N B 1, K C N D 3, K C N J 11, A B C C 9, A D R B 1$, and $S L C 18 A 2$

\begin{tabular}{|c|c|c|c|c|c|}
\hline Gene & Exon $^{\mathrm{a}}$ & Forward primer & Reverse primer & $\begin{array}{l}\text { Annealing } \\
\text { temperature } \\
\left({ }^{\circ} \mathrm{C}\right)\end{array}$ & $\begin{array}{l}\text { GenBank } \\
\text { accession } \\
\text { number }\end{array}$ \\
\hline \multirow[t]{22}{*}{ KCNB1 } & 1.1 & CTT GCC GTC GAG TGA CAG C & TCC AGG GTA CGC CAG AGT A & 58 & NT 002370 \\
\hline & 1.2 & GCA TGG CTC CCG CTC CAC & GCG AGT CGT GCG TGT TGC & 58 & NT_002370 \\
\hline & 1.3 & GCA CGC GGC TGG GCA AGC & GCT GAG CGC GCA CAT CTC & 72 & NT_002370 \\
\hline & 1.4 & CGC CTT CAC CTC CAT CCT CA & GGT CTC GGC CTC ACG CTT GA & 58 & NT_002370 \\
\hline & 1.5 & CCC GCT ACC ACC AGA AGA AAG A & CCC AGA CCA GCA GCC CCC AGA T & 58 & NT_002370 \\
\hline & 2.1 & ATC ATC CTG TGG GGT GGT TG & GTG GAC TGG CCG AAC TCA TC & 54 & NT_002370 \\
\hline & 2.2 & TCA ACA CGC TGC CTG AGC & CAC TTC TTG GGC GAG GAG & 54 & NT_002370 \\
\hline & 2.3 & CAT GGA GTA CCT GCT GAG G & GCT TAA GGA TGC GGA GAA T & 54 & NT_002370 \\
\hline & 2.4 & TCA CCG AAT CCA ACA AGA G & AGG AAG AGG ATG AGC AAG C & 58 & NT_002370 \\
\hline & 2.5 & CAG TCT CTG GGC TTC ACT TT & GTC TTG GGG TAG ATG TCT CC & 58 & NT_002370 \\
\hline & 2.6 & CCA TCA CCA TGA CTA CTG TTG & GAT TGC TTT CTC CTG TCT CTT & 58 & NT_002370 \\
\hline & 2.7 & TCC CCA TCA TCG TCA ATA ACT & ATT CTC CCC ATT TTT CTC AAC & 58 & NT_002370 \\
\hline & 2.8 & AGC ATT GAG ATG ATG GAC ATT GT & GAA GAC GAT CTG GCT TTT TCA GG & 58 & NT_002370 \\
\hline & 2.9 & CAA ATG GAA ATG GAC AAA GA & TGC TGA CTC CTT GGT ATT GA & 58 & NT_002370 \\
\hline & 2.10 & GCC AAG ACC CAA TCC CAA CC & TGA CCC CTT CTG TGC GAG TG & 62 & NT_002370 \\
\hline & 2.11 & AGC ACA GAG CAA ACC AAA GG & GCT GGG GAG TGA TGT CAA AG & 58 & NT_002370 \\
\hline & 2.12 & GCC ACC AGA TTC TCC CAC AG & GCC TCC ACA AAC CTA CCA CC & 62 & NT_002370 \\
\hline & 2.13 & ATC ACT CCC CAG CAA GAC TG & CAC CCT CCA TGA AGT TGA CT & 62 & NT_002370 \\
\hline & 2.14 & AAC TAA CAA CCC TTT GAA GC & CTG TGG TGT AGA TGG AGG AC & 62 & NT_002370 \\
\hline & 2.15 & CGC TGG ACT GGA GTG TGC & GCT GTC CCT CAT CAT CTG & 62 & NT_002370 \\
\hline & 2.16 & CAC CAG TAC ATT GAC GCA G & AAT GCT TCT GTG GAG TAA ATA C & 62 & NT_002370 \\
\hline & 2.17 & CTC CCC TTT ACC CAC CTC & GGC AGG GCA GTT CAG ATG & 62 & NT_002370 \\
\hline \multirow[t]{16}{*}{ KCND3 } & 1.1 & GGC TGC CGG CCC AAG AGC & AAC CTC CGC CCA CTC ACG & 65 & AF166009 \\
\hline & 1.2 & GAA CAA GCG GCA GGA TGA GC & TGA GCA CGC AGC GGA ACA CC & 62 & AF166009 \\
\hline & 1.3 & GGC AGC ACG GAG AAG GAG TT & CGT CGT CGT AGG CAG AGA TG & 62 & AF166009 \\
\hline & 1.4 & GTG TTC CGC TGC GTG CTC AA & TCC TGG TTG TTC TCC GAG TC & 62 & AF166009 \\
\hline & 1.5 & AGT ACA AGG ACC GCA AGA GG & GAC CGA GAC AGC GAT GAA GA & 62 & AF166009 \\
\hline & 1.6 & CAC CAG CAC GCT GGC CCT GGT C & CTC CTC TAC CCA TGG TGA C & 62 & AF166009 \\
\hline & 2.1 & CTC CCT TAC ACC CTG TTT СТC TC & GGT AAA TCC GGC TAA AGT TGG AA & 62 & AF166010 \\
\hline & 2.2 & TTG AGT GGC GTC CTG GTC ATT GC & GTG CTC CCC CGC ATC CTT TAC AC & 62 & AF166010 \\
\hline & 3 & CAT TCT TTT ATT ATC TCT GCC AAT CA & ACA AGC CCA TCT ACC CCT TTA TGT TC & 62 & AF166011 \\
\hline & 4 & CCA CCA GCT TTT TAC TCA ATC TCT & AAA GGG TCA GGG TCA GCG ATG AAA & 62 & AF166011 \\
\hline & 5 & GTA CAA TCA ATG GTG TTT TTA TCT TC & AAA GGG GAG AAT CCA CAG ACT CAG AA & 62 & AF166011 \\
\hline & 6.1 & CCT TTT CCT ACT TCT TCT CCT TCT GC & GAC AGT GAG GGA CTT CTT GTG GAT GG & 62 & AF166011 \\
\hline & 6.2 & ACC CAG GCC TCA CTA CCA C & AGG GAG GGC TGC TCA CTG C & 65 & AF166011 \\
\hline & 6.3 & CTC AGC ACG ATC CAC ATC & GTA GAG GGG CTC ATG CAT & 62 & AF166011 \\
\hline & 7.1 & CCA TCA TCA АCT CAC TCT TTT C & CTA TGG AAG GAA TGT TCG TGT T & 62 & AF166011 \\
\hline & 7.2 & CAC AGC CAT CAT CAG CAT CC & CAT TCC CCA СТA ССС АCТ СТ & 72 & AF166011 \\
\hline \multirow[t]{6}{*}{ KCNJ11 } & 1.1 & GCG AGA GGA CTC TGC AGT GA & CAC CAG GGT GT GAA CAC GT & 58 & NT_000564 \\
\hline & 1.2 & GAA AGG CAA CTG CAA CGT GG & TAG TGA CTT GGA CCT CAA TG & 58 & NT_000564 \\
\hline & 1.3 & CTG TGT CAC CAG CAT CCA GT & TGA TGA TCA TGC T G TGC GG & 58 & NT 000564 \\
\hline & 1.4 & TCA GCA AGC ATG CGG TGA TC & ACG CCT TCC AGG ATG ACG AT & 58 & NT_000564 \\
\hline & 1.5 & CTA CCA TGT CAT TGA TGC CA & GCA CTT TGA TGG TGT TGC CA & 58 & NT_000564 \\
\hline & 1.6 & CGT TAC TCT GTG GAC TAC TC & TGG GCT ACA TAC CAC ATG GT & 58 & NT_000564 \\
\hline \multirow[t]{31}{*}{$A B C C 9$} & 1 & TTT TAT GAA CAA GAG TTT AC & TCC CAA ATC TCA GCC ATT AG & 50 & AF061289 \\
\hline & 2.1 & GGA AGT GTT AAG GTT TCA GGA C & AAG AAT CCA TCT CAG GTT ATG T & 50 & AF061290 \\
\hline & 2.2 & TAC AAA TTC ACC ACA ACA CA & GGC ACA AGT TAC AAA GAT GT & 50 & AF061290 \\
\hline & 3 & ATT ATC TGG AAC CAT CAG C & GGA GAA ACA ACA AAA GTG A & 50 & AF061291 \\
\hline & 4 & TTC TCT TTT TGT CTT TTT CT & TGA ATC CAA GTA ACT AAA CA & 50 & AF061292 \\
\hline & 5 & AAT GTA GAA AAG GTT GAA AT & TGG AAG ACA GAC GCT AAA TC & 58 & AF061293 \\
\hline & 6.1 & ATT TTG TTA CAT CTG TTT TT & AAT ACA AAG AGG TCC AGC AA & 50 & AF061294 \\
\hline & 6.2 & GGC GAC CAA TTC TAC TTA GT & CCA TTG AAA TCA CCA GGA AC & 50 & AF061294 \\
\hline & 7 & TTT ATG GAT TTA CCT GGT TTG T & ATA GTA TTC ACA GCC TTT ACA T & 50 & AF061295 \\
\hline & 8.1 & TGA AAA TAA TCA TAG TGT CAA T & TGA ACA GGC ATA GCC CAT AGA T & 50 & AF061295 \\
\hline & 8.2 & ATA ATA AAA TCC TTA GGC TCT CTA C & CCT CTG CAG ACA AAA ATC TTA CTT A & 50 & AF061295 \\
\hline & 9.1 & ACC CAT TCA TCT TCT CTT TTC C & GAG CCT CTG CCA ACT TTG TAG C & 50 & AF061296 \\
\hline & 9.2 & AAT GGG CGT GAT TCT GCT CTA T & TGT TAC TTA CAA GGC ACT TTT C & 50 & AF061296 \\
\hline & 10 & GGA TGC AGC TGG TAT TAT TTT TAC T & AAA GTC GCC TGT TCT TAG AGC AAC C & 50 & AF061297 \\
\hline & 11 & TAC TCT TTG TCC TTC CTG TCT TCA A & CAC TGA CTT GGT TTC ATT TCT AAA A & 55 & AF061298 \\
\hline & 12 & TTT TTC CAG CTT TCT CTA AA & AAA ATG CCA GAC ACT TCA GT & 55 & AF061299 \\
\hline & 13 & TCT TAT TCT GTG TTT ATT GTT TTT & CTG CTG AGA CTG TCC TCT ATT CAC & 55 & AF061300 \\
\hline & 14.1 & AAG CCT CAA CTT GTG TTC TTT TCC AT & TTT ATT GCA ATG TCC TCT GTT TCT GC & 50 & AF061301 \\
\hline & 14.2 & CTA TGA GCA ATC AAC ACG GCG TCT ACG & TGG GAC ACT TTC ACA GAA CTT CAC CAT & 50 & AF061301 \\
\hline & 15 & GGT CTC CCA AAG TGC TGT GAT TAC A & CTT GGA AAA CTA TGG TTA CGG TCA T & 55 & AF061302 \\
\hline & 16 & TCT GTA GTT ATC CTT TGT TT & TAT GAC ATA GCA ATG GAA GC & 55 & AF061303 \\
\hline & 17 & GTG TTT ATT GTA TCT CTT ACC TGA CG & TCT GAG GAA GGA AGT TAT TCT TAT TA & 55 & AF061304 \\
\hline & 18 & GTA CCT TTG CAT AAT CCT GGT GTT TT & AAA GAG TTG CCA TTT TCG GTT CTA AA & 55 & AF061305 \\
\hline & 19 & ATG GAC AAT GTA TTA CTT GAG AT & GAT TAA AGA CAA CCA GAA GAC TT & 50 & AF061306 \\
\hline & 20 & AAT GGG TAT TTG GGG GTG TCT CT & TTC CTT GAG TTA CTT GAC TTA CA & 55 & AF061307 \\
\hline & 21 & TCC ATC TTT CCT CCA TTT TA & GAA CTA CCT TTA TTT CAG AA & 55 & AF061308 \\
\hline & 22 & CTT TTC GTC ACA TTT CTG GGA ATA & CTC TTT TGA ACA TTG GGC TAT GAA & 55 & AF061308 \\
\hline & 23 & TAA GTC ACA ACC TAT TGG TGT TAG C & TCG CAT CCT GTT ATC CCA TTA GAA T & 55 & AF061309 \\
\hline & 24.1 & CTG AAG CTT AAA CAA AAG TT & CAG GAT GAG CAG GAA GAA TC & 50 & AF061310 \\
\hline & 24.2 & AAA TGC CAT GGA AAA CCT G & AAA ACA AAA CCG AAC CAA T & 50 & AF061310 \\
\hline & 25 & TAT GTG CTT TTT GTG TTG GT & ATG TGT GGA TGA GAA ATA AC & 50 & AF061311 \\
\hline
\end{tabular}


Table 1. Continued

\begin{tabular}{|c|c|c|c|c|c|}
\hline Gene & Exon $^{\mathrm{a}}$ & Forward primer & Reverse primer & $\begin{array}{l}\text { Annealing } \\
\text { temperature } \\
\left({ }^{\circ} \mathrm{C}\right)\end{array}$ & $\begin{array}{l}\text { GenBank } \\
\text { accession } \\
\text { number }\end{array}$ \\
\hline & 26 & TTG ATC ATA TAC TTC CAT CTG TT & ATT TAG CTT ACA TTG TGA AAC TA & 55 & AF061311 \\
\hline & 27 & GCT AAT CAT ATT TTC CTT TT & AGG GCT GAC TGT AGA TAA GC & 55 & AF061312 \\
\hline & 28 & TAG TTT TTG AGT CTG GAT GC & TTT GTT GAT GAT GTT ATT GC & 55 & AF061313 \\
\hline & 29 & GTT TCT TAT TAC CTC TCC TG & TTA CAA CTG TCT GCC TCT TT & 55 & AF061314 \\
\hline & 30 & CTT TTA ACA TTT TCT CTT GCT TT & ATT CAA TTC CCA TTT ATG AGT GA & 55 & AF061315 \\
\hline & 31 & TGG AGT GGT GAA CAT CGT GT & GTC TTC TCT ATT TGG TTT CC & 55 & AF061316 \\
\hline & 31 & TGG AGT GGT GAA CAT CGT GT & GTC TTC TCT ATT TGG TTT CC & 50 & AF061316 \\
\hline & 32 & CTG CCT ATT ATC TGT CTA TTT C & CTT GGC TGG GAA GTA TGA AGA G & 50 & AF061317 \\
\hline & 33 & TCT CTT TGA GTG ACA CCT GTT & TTA AAG GAA AGC ACC AAG TGG & 50 & AF061318 \\
\hline & 34 & ACC CCT AAC TAA GAA ATA ACT TT & AGG CAT ACA GGT GCT GCT AAA TA & 50 & AF061319 \\
\hline & 35.1 & CAA AAC AGA ATG GCA CAT ACA T & GCA TAA AAC GTA AGA TAG ACA T & 50 & AF061320 \\
\hline & 35.2 & ACC TAA TTA TTC CCT ACA GAT TT & GCA TAA AAC GTA AGA TAG ACA TA & 50 & AF061320 \\
\hline & 36 & CTT GCT TCT TTC TCT GAT TTA G & TTT TCA TGC ACA TTT CTC CAA T & 50 & AF061321 \\
\hline & 37 & TGA CAA AGT AAT CCC GAG AAA A & GAG GTA TAC CAA CTC CGT CTT C & 50 & AF061322 \\
\hline & 38 & CAT TTT TCC TTC TGC TGA TTC T & TTA GCC ATG CCT TAC TGA GAG G & 50 & AF061323 \\
\hline \multirow[t]{12}{*}{$A D R B 1$} & 1.1 & CGG GCT TCT GGG GTG TTC C & GAC GCG GGC ACC AGC AGC & $65-62 \mathrm{TD}^{\mathrm{b}}$ & NM000684 \\
\hline & 1.2 & CCG CAC CGC TCC CCG ACG & GCC CGC CAC GAT GAG CAG CAC & $70-67 \mathrm{TD}^{\mathrm{b}}$ & NM000684 \\
\hline & 1.3 & ATG GGT CTG CTG ATG GCG & GTA CTC CCA GCG GCC CC & $65-62 \mathrm{TD}^{\mathrm{b}}$ & NM000684 \\
\hline & 1.4 & CCG TTC GGG GCC ACC ATC GT & GCG CGT CAG CAG GCT CTG GTA G & $72-69 \mathrm{TD}^{\mathrm{b}}$ & NM000684 \\
\hline & 1.5 & CGC TAC CTC GCC ATC ACC TCG C & CGG TTG GTG ACG AAG TCG CAG C & $71-68 \mathrm{TD}^{\mathrm{b}}$ & NM000684 \\
\hline & 1.6 & CGC CGC TGC TAC AAC GAC CC & TGG GCC GCC GAG GAA ACG & $70-67 \mathrm{TD}^{\mathrm{b}}$ & NM000684 \\
\hline & 1.7 & GCC CAG AAG CAG GTG AAG AAG ATC G & GCC GCC GCT TAC CCG CAC & $65-62 \mathrm{TD}^{\mathrm{b}}$ & NM000684 \\
\hline & 1.8 & CTC GCC CTC GCC CGT CCC & GCA GCC AGC AGA GCG TGA AGA C & $72^{\mathrm{c}}$ & NM000684 \\
\hline & 1.9 & CGC TCA AGA CGC TGG GCA TCA T & CGG AAG TCG GGG CTG CGG & $65-62 \mathrm{TD}^{\mathrm{b}}$ & NM000684 \\
\hline & 1.10 & CAA CTC GGC CTT CAA CCC CAT C & ATC GTC GTC GTC GTC GTC CG & $68-65 \mathrm{TD}^{\mathrm{b}}$ & NM000684 \\
\hline & 1.11 & GAC CCC CGC CAT CGC CCG & CGG GGC GGC ACG GCT CG & $72-69 \mathrm{TD}^{\mathrm{b}}$ & NM000684 \\
\hline & 1.12 & GGC GGC GGA CAG CGA CTC & TGA TTC AGA CGA GGA TTG TGG GC & $68-65 \mathrm{TD}^{\mathrm{b}}$ & NM000684 \\
\hline \multirow[t]{19}{*}{ SLC18A2 } & 1 & TAA ACT GAG CGG CGG CGG & ACC GGG GCC GTG TTA CCT C & 62 & NT_000545 \\
\hline & 2 & CCT GGG GGA CGG GAG AAT G & CAG CCA GCG GAC CAG CG & 62 & NT_000545 \\
\hline & 3.1 & GTG ATC CAC CTG CCT CAG C & TCT CAT GCT TAA TGC TGT ACA GAT & 62 & NT_000545 \\
\hline & 3.2 & ACC ACC TTG CCA TTC TGC TCT TAT C & TCA GGT CTC TGG TAG CAT TCC CG & 62 & NT_000545 \\
\hline & 3.3 & GCC TCC ATC TCA GAC AGC & AGG AGG TCT TTG TCT TCA CTG & 64 & NT_000545 \\
\hline & 4 & AAG GGT AGC CAG GCG AAA ATC A & TCA ATG CCC ACC CTG ATG AGA C & 56 & NT_000545 \\
\hline & 5 & ACG GTT GTG TCC CAG CAG C & GCC CCT CAC GAA CTC ACT CA & 64 & NT_000545 \\
\hline & 6 & GGG AAG GCG AGT CAC GC & GGA CTG CCG CTG TCC AGC & 58 & NT_000545 \\
\hline & 7 & CTA GTA CAG GGA GAG GGC ATG T & AAG GTC ATG GCA TGG CG & 62 & NT_000545 \\
\hline & 8 & TGC TTG GGC CAC ACC TGA TT & CCC TCA TTC CCA GCC GCT & 58 & NT_000545 \\
\hline & 9 & GAG GTA AGC GGC TGG GAA TGA G & CCT CGC ATT CCC CTG ACT CA & 58 & NT_000545 \\
\hline & 10 & TGG AAA TGA GAG AGG AGG CAG & AGA AGA GCC CAC CCC AGT C & 62 & NT_000545 \\
\hline & 11 & TTA CTT TTT CTA ACA TAT GAC TGA T & TGA GTG TTG TTT TCA TAT CAT CT & 58 & NT_000545 \\
\hline & 12 & TTG GAC TAG AAA AAA ATC AAA TGA TC & CAC AGA TTG TTC CCA CTT GTC G & 62 & NT_000545 \\
\hline & 13 & GAG TTG GAT CTC TTG TTA TTT TAG CA & TAA ATG TAG GCA CAA GGT TCG T & 58 & NT_000545 \\
\hline & 14 & CTA TAC ACT GTG TTC CCT GAC TGT C & GGT TCT TAT GAA AAG CCA ATG TC & 62 & NT_000545 \\
\hline & 15 & GCT TTA TCT AAT TCT CTG TTT CC & TTA TCA AGA AAT GCT CAC TGC & 62 & NT_000545 \\
\hline & 16.1 & AGA AGT TAA TAT ACT TGC ACT TTG C & CTG TTT TAT ACA ATT AAA CAC TTT GAT G & 58 & NT_000545 \\
\hline & 16.2 & ATG AAG AAT CTG AAA GTG ACT GAG & AAG GCT GTT GGC AAT CG & 62 & NT_000545 \\
\hline
\end{tabular}

SSCP, Single-strand conformation polymorphism

${ }^{a}$ Exons followed by dots and sequential numbers were subdivided for SSCP analysis

${ }^{\mathrm{b}} \mathrm{TD}$, indicates touch-down polymerase chain reaction (PCR). ${ }^{\mathrm{c}}$ Shuttle PCR was used

patients and 150 normal controls. Our screening method was described previously (Itoh et al. 1998). In brief, we prepared genomic DNA from blood samples according to standard protocols, after obtaining written informed consent from each participant. All exons of each gene being scrutinized (KCNB1, KCND3, KCNJ11, ABCC9, ADRB1, and $S L C 18 A 2$ ), as well as flanking intronic sequences, were amplified by newly designed polymerase chain reaction (PCR) primers (Table 1) and subjected to single-strand conformation polymorphism (SSCP) analysis. Fragments presenting aberrant conformers were sequenced with $\mathrm{ABI}$ 3700 instruments (Applied Biosystems, Foster City, CA, USA).

To investigate the allelic frequencies for each SNP in our normal control population, we hybridized allele-specific oligonucleotides to DNA from 150 normal, unrelated individuals in the manner described by Saiki et al. (1986). An oligonucleotide specific for each allele was synthesized to discriminate between alleles at each polymorphic site.

\section{Results and discussion}

In all, we confirmed 20 SNPs ( 2 in $K C N B 1,3$ in $K C N D 3$, 3 in KCNJ11, 7 in $A B C C 9,3$ in $A D R B 1$, and 2 in $S L C 18 A 2$ ), and we examined the frequency of each allele in the Japanese population. Table 2 summarizes the results.

Six variations were detected in one of the LQTS patients (Table 2). Because none of these would change an amino acid, and because none would seem to lead to splicing abnormalities, judging from their surrounding sequences, at 
Table 2. SNPs of six candidate genes for long QT syndrome

\begin{tabular}{|c|c|c|c|c|c|c|}
\hline Gene & $\begin{array}{l}\text { Nucleotide } \\
\text { change }^{\mathrm{a}}\end{array}$ & $\begin{array}{l}\text { Amino acid } \\
\text { change }\end{array}$ & Region & $\begin{array}{l}\text { Frequency } \\
\text { of minor } \\
\text { allele }\end{array}$ & $\begin{array}{l}\text { Number of } \\
\text { chromosomes } \\
\text { examined }\end{array}$ & Previous report (if any) \\
\hline \multirow[t]{2}{*}{$K C N B 1$} & $1071 \mathrm{C}>\mathrm{T}$ & D357D & Exon 2 & 0.01 & 100 & \\
\hline & $768 \mathrm{G}>\mathrm{A}$ & $\mathrm{K} 256 \mathrm{~K}$ & Exon 2 & Rare $^{\mathrm{b}}$ & 188 & \\
\hline \multirow[t]{3}{*}{$K C N D 3$} & $375 \mathrm{G}>\mathrm{A}$ & $\mathrm{P} 125 \mathrm{P}$ & Exon 1 & 0.22 & 100 & \\
\hline & $1106+15 \mathrm{G}>\mathrm{A}$ & Intronic variant & Intron 1 & Rare $^{\mathrm{b}}$ & 188 & \\
\hline & $1269+15 \mathrm{C}>\mathrm{A}$ & Intronic variant & Intron 2 & 0.22 & 100 & \\
\hline \multirow{3}{*}{ KCNJ11 } & $67 \mathrm{G}>\mathrm{A}$ & E23K & Exon 1 & 0.38 & 100 & Hani et al. 1998; Sakura et al. 1996 \\
\hline & $570 \mathrm{~T}>\mathrm{C}$ & A190A & Exon 1 & 0.35 & 100 & Sakura et al. 1996 \\
\hline & $1009 \mathrm{~A}>\mathrm{G}$ & $\mathrm{I} 337 \mathrm{~V}$ & Exon 1 & 0.38 & 100 & Hani et al. 1998; Sakura et al. 1996 \\
\hline \multirow[t]{7}{*}{$A B C C 9$} & $142+47 \mathrm{C}>\mathrm{T}$ & Intronic variant & Intron 1 & Rare $^{b}$ & 188 & \\
\hline & $285-22 \mathrm{G}>\mathrm{T}$ & Intronic variant & Intron 2 & 0.03 & 100 & \\
\hline & $1012-59 \mathrm{~T}>\mathrm{C}$ & Intronic variant & Intron 6 & 0.26 & 100 & \\
\hline & $1660-65 \mathrm{C}>\mathrm{T}$ & Intronic variant & Intron 11 & 0.26 & 100 & \\
\hline & $2397+58 \mathrm{~A}>\mathrm{T}$ & Intronic variant & Intron 20 & Rare $^{\mathrm{b}}$ & 188 & \\
\hline & $3561+4 \mathrm{C}>\mathrm{G}$ & Intronic variant & Intron 29 & Rare $^{\mathrm{b}}$ & 188 & \\
\hline & $4488 \mathrm{~T}>\mathrm{C}$ & $\mathrm{F} 1496 \mathrm{~F}$ & Exon 36 & Rare $^{\mathrm{b}}$ & 188 & \\
\hline \multirow[t]{3}{*}{$A D R B 1$} & $145 \mathrm{~A}>\mathrm{G}$ & S49G & Exon 1 & 0.17 & 100 & Maqbool et al. 1999; Podlowski et al. 2000 \\
\hline & $1071 \mathrm{C}>\mathrm{T}$ & R357R & Exon 1 & 0.02 & 100 & \\
\hline & $1165 \mathrm{C}>\mathrm{G}$ & $\mathrm{R} 389 \mathrm{G}$ & Exon 1 & 0.15 & 100 & Maqbool et al. 1999; Tesson et al. 1999 \\
\hline \multirow[t]{2}{*}{$S L C 18 A 2$} & $813+15 \mathrm{~T}>\mathrm{C}$ & Intronic variant & Intron 6 & 0.30 & 100 & \\
\hline & $688 \mathrm{~A}>\mathrm{T}$ & M230L & Exon 6 & 0.070 & 300 & \\
\hline
\end{tabular}

SNP, Single-nucleotide polymorphism

${ }^{\text {a }}$ Nucleotide numbering starts from ATG start codon

${ }^{\mathrm{b}}$ Indicates that the allele was not identified in normal controls

present, we are considering all six to be rare polymorphisms.

Of the 20 different SNPs detected in this study, 5 had been reported already, and their allelic frequencies had been determined in Caucasian populations (Sakura et al. 1996; Hani et al. 1998; Maqbool et al. 1999; Tesson et al. 1999; Podlowski et al. 2000). We found no apparent differences in allelic frequencies for these five SNPs between Caucasian and Japanese populations.

We believe the data reported here will provide useful information for association studies designed to identify genes related to nonfamilial arrhythmias.

Acknowledgments This work was supported in part by a Grant-in-Aid from the Ministry of Education, Science, Sports, and Culture of Japan. We are grateful to Kaori Sato for secretarial assistance.

\section{References}

Abbott GW, Sesti F, Splawski I, Buck ME, Lehmann MH, Timothy KW, Keating MT, Goldstein SA (1999) MiRP1 forms $\mathrm{I}_{\mathrm{Kr}}$ potassium channels with $H E R G$ and is associated with cardiac arrhythmia. Cell 97:175-187

Barry DM, Xu H, Schuessler RB, Nerbonne JM (1998) Functional knockout of the transient outward current, long-QT syndrome, and cardiac remodeling in mice expressing a dominant-negative $\mathrm{Kv} 4$ alpha subunit. Circ Res 83:560-567

Bennett PB, Yazawa K, Makita N, George AL Jr (1995) Molecular mechanism for an inherited cardiac arrhythmia. Nature 376:683685

Bhandari AK, Scheinman MM, Morady F, Svinarich J, Mason J, Winkle R (1984) Efficacy of left cardiac sympathectomy in the treatment of patients with the long QT syndrome. Circulation 70:10181023

Curran ME, Splawski I, Timothy KW, Vincent GM, Green ED, Keating MT (1995) Molecular basis for cardiac arrhythmia: HERG mutations cause long QT syndrome. Cell 80:795-803
Fujimoto Y, Morita H, Fukushima KK, Ohe T (1999) Nicorandil abolished repolarization alternans in a patient with idiopathic long QT syndrome. Heart 82:8

Hani EH, Boutin P, Durand E, Inoue H, Permutt MA, Velho G, Froguel P (1998) Missense mutations in the pancreatic islet beta cell inwardly rectifying $\mathrm{K}^{+}$channel gene $(K I R 6.2 / B I R)$ : a meta-analysis suggests a role in the polygenic basis of type II diabetes mellitus in Caucasians. Diabetologia 41:1511-1515

Hiraoka M, Fan Z (1989) Activation of ATP-sensitive outward K current by nicorandil (2-nicotinamidoethyl nitrate) in isolated ventricular myocytes. J Pharmacol Exp Ther 250:278-285

Itoh T, Tanaka T, Nagai R, Kikuchi K, Ogawa S, Okada S, Yamagata S, Yano K, Yazaki Y, Nakamura Y (1998) Genomic organization and mutational analysis of $K V L Q T 1$, a gene responsible for familial long QT syndrome. Hum Genet 103:290-294

Itokawa K, Sora I, Schindler CW, Itokawa M, Takahashi N, Uhl GR (1999) Heterozygous VMAT2 knockout mice display prolonged QT intervals: possible contributions to sudden death. Brain Res Mol Brain Res 71:354-357

Maqbool A, Hall AS, Ball SG, Balmforth AJ (1999) Common polymorphisms of beta1-adrenoceptor: identification and rapid screening assay. Lancet 353:897

Noma A (1983) ATP-regulated $\mathrm{K}^{+}$channels in cardiac muscle. Nature 305:147-148

Podlowski S, Wenzel K, Luther HP, Muller J, Bramlage P, Baumann G, Felix SB, Speer A, Hetzer R, Kopke K, Hoehe MR, Vallukat G (2000) $\beta 1$-Adrenoceptor gene variations: a role in idiopathic dilated cardiomyopathy? J Mol Med 78:87-93

Saiki RK, Bugawan TL, Horn GT, Mullis KB, Erlich HA (1986) Analysis of enzymatically amplified beta-globin and HLA-DQ alpha DNA with allele-specific oligonucleotide probes. Nature 324:163166

Sakura H, Wat N, Horton V, Millns H, Turner RC, Ashcroft FM (1996) Sequence variations in the human Kir6.2 gene, a subunit of the betacell ATP-sensitive K-channel: no association with NIDDM in white Caucasian subjects or evidence of abnormal function when expressed in vitro. Diabetologia 39:1233-1236

Splawski I, Tristani-Firouzi M, Lehmann MH, Sanguinetti MC, Keating MT (1997) Mutations in the hminK gene cause long QT syndrome and suppress $\mathrm{I}_{\mathrm{Ks}}$ function. Nat Genet 17:338-340

Tesson F, Charron P, Peuchmaurd M, Nicaud V, Cambien F, Tiret L, Poirier O, Desnos M, Jullieres Y, Amouyel P, Roizes G, Dorent R, Schwartz K, Komajda M (1999) Characterization of a unique genetic 
variant in the beta1-adrenoceptor gene and evaluation of its role in idiopathic dilated cardiomyopathy. CARDIGENE Group. J Mol Cell Cardiol 31:1025-1032

Wang Q, Li Z, Shen J, Keating MT (1996a) Genomic organization of the human SCN5A gene encoding the cardiac sodium channel. Genomics 34:9-16

Wang Q, Curran ME, Splawski I, Burn TC, Millholland JM, VanRaay TJ, Shen J, Timothy KW, Vincent GM, de Jager T, Schwartz PJ,
Towbin JA, Moss AJ, Atkinson DL, Landes GM, Connors TD, Keating MT (1996b) Positional cloning of a novel potassium channel gene: $K V L Q T 1$ mutations cause cardiac arrhythmias. Nat Genet 12:17-23

Xu H, Barry DM, Li H, Brunet S, Guo W, Nerbonne JM (1999) Attenuation of the slow component of delayed rectification, action potential prolongation, and triggered activity in mice expressing a dominant-negative Kv2 alpha subunit. Circ Res 85:623-633 